\title{
Determination of the Scattering Amplitude from the Differential Cross-Section and Unitarity*
}

\author{
D. Atkinson \\ Theoretical Institute of the University, Groningen, Netherlands \\ P. W. Johnson \\ Illinois Institute of Technology, Chicago, Illinois, USA \\ R. L. Warnock ${ }^{\star}$ \\ Physikalisches Institut der Universität Bonn, \\ and \\ Department of Physics, Imperial College, London, U.K.
}

Received June 2, 1972

\begin{abstract}
When the differential cross-section for spin-zero elastic scattering is given, the elastic unitarity condition constitutes a nonlinear integral equation for the phase of the scattering amplitude. Existence and uniquences theorems for solutions of the equation were obtained by Newton and Martin. Some improvements of the Newton-Martin results on uniqueness and iterative construction of solutions are obtained. Certain details of rigour in the applications of Schauder's theorem by Newton and by Martin are supplied. The case of inelastic spin-zero scattering is treated by adding a term to the unitarity condition to account for absorption. It is shown that in the inelastic region one may have infinitely many different scattering amplitudes with a given differential cross-section. This result is potentially important in phase-shift analysis, since it means that there is a "continuum ambiguity" in the determination of phases and elasticities from scattering data.
\end{abstract}

\section{Introduction}

A fundamental question of scattering theory is this: to what extent is the scattering amplitude determined when the differential cross-section is given and the unitarity condition is imposed? The problem is interesting in connection with phase-shift analysis, as well as in epistomological questions of quantum theory. For the case of spin-zero elastic scattering, a partial answer was provided by Crichton [1], Newton [2], Martin [3], and others [4]. Newton and Martin were the first to obtain sufficient conditions for existence and uniqueness of an elastic amplitude with a given cross-section.

* Work supported in part by the National Science Foundation and a NATO Research Grant.

$\star \star$ Address after July 1, 1972: Illinois Institute of Technology, Chicago, Illinois 60616. 
The elastic unitarity equation is a nonlinear integral equation for the phase of the scattering amplitude, when the modulus (i.e. the differential cross-section) is given. The problem, then, is to investigate solutions of this integral equation.

The Newton-Martin conditions for existence and uniqueness of solutions are stated in terms of the following functional of the differential cross-section:

$$
\begin{aligned}
\sin \mu= & \sup _{-1 \leqq x \leqq 1} \frac{1}{4 \pi g(x)} \int_{-1}^{1} d y \int_{0}^{2 \pi} d w g\{y\} \\
& \cdot g\left\{x y+\left[\left(1-x^{2}\right)\left(1-y^{2}\right)\right]^{\frac{1}{2}} \cos w\right\},
\end{aligned}
$$

where $x$ is the cosine of the scattering angle and

$$
g(x)=k(d \sigma / d \Omega)^{\frac{1}{2}},
$$

$k$ being the momentum and $d \sigma / d \Omega$ the differential cross-section. Newton and Martin obtained the inequality $\sin \mu<1$ as a sufficient condition for existence of at least one continuous solution for the phase of the amplitude, $\varphi(x)$. We have noticed, however, that the proofs given in Refs. [2] and [3] are incomplete, but that they can be completed.

By an application of the contraction mapping principle, Newton showed that there is a unique continuous solution if $\sin \mu<(5)^{-\frac{1}{2}}$, and that this solution may be obtained by iterating the equation. Martin proved that there cannot be more than one solution if $\sin \mu<\sin \mu_{1} \cong 0.79$, but he did not prove convergence of the iterative sequence when $\sin \mu$ is in this range. We find that the contraction mapping principle can be applied for $\sin \mu<\sin \mu_{1}$, provided one introduces a new metric. Thus, for $\sin \mu<\sin \mu_{1}$, there exists a unique continuous solution which may be obtained by iteration.

Next we account for inelasticity by adding a term to the unitarity condition that represents absorption by other channels. Under suitable restrictions, we prove existence and uniqueness of solutions of the modified equation, assuming that the inelastic term is given. By means of the implicit function theorem, we show that the solution $\varphi$ varies continuously when the inelastic term is varied continuously. (This is true at least when the differential cross-section and inelastic term are such that the contraction mapping principle applies: in other cases the question is not settled.) This means that one can find a continuum of values of the phase-shifts $\delta_{l}$ and elasticities $\eta_{l}$, all corresponding to the same differential cross-section. The continuum ambiguity remains even if one includes the constraint of a fixed total cross-section, via the optical theorem.

These remarks indicate an essential difficulty of phase-shift analysis in the inelastic region. The existence of a continuum of solutions (even 
when experimental errors are assumed to be zero) has not been generally recognized, since one always truncates the partial-wave series. The truncation yields a finite number of minima of chi-squared in the phaseshift fitting programme, so that one obtains only a finite number of different phase-shifts. The existence of a continuum of solutions depends essentially on keeping an infinite number of partial waves. The existence of a continuum ambiguity in the inelastic region was recognized by Bowcock and Hodgson [4], who constructed a class of amplitudes with the same modulus but different phases, all satisfying the constraint of elastic unitarity. Our discussion is more general, since it applies to a wide class of differential cross-sections, and it does not rely on a particular model.

We do not obtain a useful estimate of the amount by which the inelastic term may be varied when the differential cross-section is fixed. This question should be investigated numerically in specific cases [5]. One should also do numerical calculations for the régime in which our analytical arguments do not succeed [5] (e.g. when the differential crosssection or the inelasticity is too large).

Since energy-independent phase-shift analysis is faced with serious ambiguities in the inelastic region, it is natural to enquire about the prospects for energy-dependent analysis. The theorems of Newton and Martin show that the latter is indeed possible, in principle, if one assumes that the scattering amplitude is analytic in the energy $E$ (in the usual cut plane) at each $x=\cos \vartheta$. If the scattering lengths are finite, the function $g(x)$ vanishes uniformly as the energy approaches threshold from above, which means that $\sin \mu$ also vanishes in that limit. It follows that $\sin \mu<\sin \mu_{1}$ in some energy interval $E_{0} \leqq E \leqq E_{1}$, where $E_{0}$ is the threshold energy, and $E_{1}$ is less than the threshold for inelastic processes. Hence, for $E_{0} \leqq E \leqq E_{1}$, we have a unique, continuous, unitary amplitude with a given differential cross-section. The amplitude is then determined at all $E$ by analytic continuation, provided the differential cross-section is known in any subinterval of $\left[E_{0}, E_{1}\right]$. As a matter of principle, it would then be superfluous to perform energy-independent phase-shift analyses in the inelastic region. Needless to say, these considerations are of very little help in practical cases, because of the difficulty of making a stable analytic continuation. Perhaps it is a useful reminder, however, of the potential power of analyticity to reduce the ambiguites of scattering phenomenology.

In Section 2, we define the notation and then study the integral equation by means of the contraction mapping principle. We first employ a Banach space of continuous functions with supremum norm, as in Ref. [2], but obtain a slightly weaker condition for a contraction mapping than that of Ref. [2]; viz., $\sin \mu<\sin \mu_{0} \cong 0.62$ in place of $\sin \mu<(5)^{-\frac{1}{2}}$ 
$\cong 0.45$. We then go over to a weighted $L^{2}$ norm, which yields a contraction mapping for $\sin \mu<\sin \mu_{1} \cong 0.79$. This gives uniqueness of the solution for $\sin \mu<\sin \mu_{1}$, (as in Ref. [3], but with a simpler proof), and also furnishes a construction of the solution by iteration. The Appendix generalizes the discussion based on the supremum norm, by taking account of a lower bound for the integral which appears in Eq. (1.1). The existence and uniqueness theorem proved in the Appendix represents a significant improvement on those of Section 2 in the case where $g(x)$ is a slowly varying function. In Section 3, we discuss the difficulty of applying Schauder's fixed-point theorem to the unitarity equation. By means of an explicit example, we show that the unitarity operator does not map bounded sets in a Banach space $S$ (consisting of continuous functions with supremum norm) into compact subsets thereof. For this reason, Schauder's theorem is not immediately applicable. In Sections 4 and 5 we work with a simple modification of the unitarity operator, which does map a particular bounded, closed, convex set into a compact subset of itself. The equation based on this operator is equivalent to the original equation, so that we obtain an existence theorem via Schauder's principle. In Section 4, the proof of compactness is carried out by a partial wave method. This was suggested by Martin, but we take care of some convergence questions which were left open previously. In Section 5 , the compactness proof is done by a new method which emphasizes geometry, and which gives as a by-product a result on continuity of solutions which is needed for the work of Section 2. Section 6 deals with the inelastic case. Fixed point theorems are applied in much the same way as in the previous sections. Only the supremum norm is used here, since the method based on the $L^{2}$ norm becomes quite awkward in the inelastic case. The implicit function theorem is applied, under conditions the same as those required for the contraction mapping principle, in order to obtain the above-mentioned results on inelastic phase-shift analysis. The interesting question of what happens when the implicit function theorem is not applicable (i.e. when the Fréchet derivative of the unitarity operator becomes singular) is left to future analysis.

\section{Notation and Existence Proof by the Contraction Mapping Principle}

We write the elastic scattering amplitude for spinless particles at a fixed energy as follows:

$$
f(x)=g(x) e^{\imath \varphi(x)},
$$

where $g=|f|$ and $x=\cos \vartheta$ is the cosine of the scattering angle. The amplitude is normalized so that the differential cross-section is

$$
\frac{d \sigma}{d \Omega}=\left[\frac{g(x)}{k}\right]^{2},
$$


$k$ being the momentum in the centre-of-mass frame. We suppose that $d \sigma / d \Omega$ is given, and that the energy lies below the first inelastic threshold. Then the unitarity condition is an integral equation for $\varphi$; namely,

where

$$
\sin \varphi(x) g(x)=\frac{1}{4 \pi} \int_{-1}^{1} d y \int_{0}^{2 \pi} d w g(y) g(z) \cos [\varphi(y)-\varphi(z)],
$$

$$
z=x y+\left[\left(1-x^{2}\right)\left(1-y^{2}\right)\right]^{\frac{1}{2}} \cos w .
$$

For some purposes, it is convenient to make a change of variable, so. that the equation takes the following form:

$$
\begin{aligned}
\sin \varphi(x) & =\iint H(x, y, z) \cos [\varphi(y)-\varphi(z)] d y d z, \\
H(x, y, z) & =\frac{g(y) g(z)}{2 \pi g(x)\left[1-x^{2}-y^{2}-z^{2}+2 x y z\right]^{\frac{1}{2}}} .
\end{aligned}
$$

The integration is over the region where the argument of the squareroot is positive. Let $\bar{B}(x ; \varphi)$ or $\bar{B}(\varphi)$ denote the right-hand side of (2.4), so that the equation may be written as

$$
\varphi=A(\varphi),
$$

with $A=\sin ^{-1} B$. We note that if $\varphi(x)$ solves (2.6), then so does $\psi(x)$ $=\pi-\varphi(x)$, as is well known. This corresponds to changing the sign of all of the phase-shifts, which does not affect the differential cross-section.

Initially, we look for solutions of (2.6) in the Banach space $S$ consisting of all real continuous functions $\varphi$ on $[-1,1]$ with the norm

$$
\|\varphi\|=\sup _{-1 \leqq x \leqq 1}|\varphi(x)| .
$$

We are interested in a subset $T$ of $S$ defined as

$$
T=\{\varphi: \varphi \in S, 0 \leqq \varphi \leqq \mu\},
$$

where $\sin \mu$ is the following functional of the differential cross-section:

$$
\sin \mu=\sup _{-1 \leqq x \leqq 1} \iint H(x, y, z) d y d z .
$$

We suppose that $g(x)$ is continuous and non-vanishing on $[-1,1]$, so that $\sin \mu$ is finite. The operator $A$ maps $T$ into itself, provided $\sin \mu \leqq 1$. This is clear from (2.2) and (2.8), if we note that the cosine in (2.2) is non-negative if $\varphi \in T$.

We shall now show that $A$ is a contraction mapping of $T$ into itself under the further restriction

$$
2 \sin \mu \tan \mu<1 \text {; i.e. } \sin \mu<\sin \mu_{0} \cong 0.62 .
$$


That is, we show that

$$
\begin{gathered}
\left\|A\left(\varphi_{1}\right)-A\left(\varphi_{2}\right)\right\| \leqq \beta\left\|\varphi_{1}-\varphi_{2}\right\|, \\
0<\beta<1, \quad \varphi_{1}, \varphi_{2} \in T .
\end{gathered}
$$

By the contraction mapping theorem [6], it then follows that there is a unique solution of (2.6) in $T$, which is the limit of the sequence $\left\{\varphi_{n}\right\}$,

$$
\varphi_{n+1}=A\left(\varphi_{n}\right),
$$

$\varphi_{0}$ being an arbitrary element of $T$.

To establish (2.11), it is convenient to use the mean-value theorem in the form [6]

$$
\left\|A\left(\varphi_{1}\right)-A\left(\varphi_{2}\right)\right\| \leqq \sup _{0 \leqq t \leqq 1}\left\|A^{\prime}\left(t \varphi_{1}+(1-t) \varphi_{2}\right)\right\| \cdot\left\|\varphi_{1}-\varphi_{2}\right\|,
$$

where $A^{\prime}(\varphi)$ denotes the Fréchet derivative of $A$, and the operator norm is

$$
\|F\|=\sup _{\substack{\varphi \in S \\ \varphi \neq 0}} \frac{\|F(\varphi)\|}{\|\varphi\|} .
$$

Eq. (2.13) holds for any operator $A$ which is continuously Fréchetdifferentiable on $\left\{\varphi: \varphi=t \varphi_{1}+(1-t) \varphi_{2}, 0 \leqq t \leqq 1\right\}$. Note that a member of this latter set belongs to $T$ when $\varphi_{1}$ and $\varphi_{2}$ do (i.e. $T$ is convex). Thus, we have to show that $\left\|A^{\prime}(\varphi)\right\|<1$ when $\varphi \in T$, where $A^{\prime}$ is defined by

$$
\begin{aligned}
A^{\prime}(\varphi) h= & -\left(1-B^{2}(\varphi)\right)^{-\frac{1}{2}} \iint H(x, y, z) \\
& \cdot \sin [\varphi(y)-\varphi(z)][h(y)-h(z)] d y d z .
\end{aligned}
$$

It is easy to check that $A^{\prime}(\varphi)$ is continuous for $\varphi \in T$, provided $\sin \mu<1$. Since $|B(x ; \varphi)| \leqq \sin \mu$ and $|\sin [\varphi(y)-\varphi(z)]| \leqq \sin \mu$, we have from (2.9) and (2.15) that

$$
\left\|A^{\prime}(\varphi) h\right\| \leqq \frac{2 \sin ^{2} \mu}{\left(1-\sin ^{2} \mu\right)^{\frac{1}{2}}}\|h\|, \quad h \in S, \varphi \in T .
$$

The condition (2.10) for existence of a unique solution in $T$ follows. From the analysis of Section 4, we also have a bound analogous to (4.22) on the variation of the solution. Hence we have

Theorem 1. If $g(x)$ is continuous on $[-1,1]$ and $\sin \mu<\sin \mu_{0} \cong 0.62$, there is a unique continuous solution $\varphi$ for which $0 \leqq \varphi \leqq \mu$. This solution is the limit of the sequence $\left\{\varphi_{n}\right\}$, where $\varphi_{n+1}=A\left(\varphi_{n}\right)$, and $\varphi_{0}$ is any continuous function such that $0 \leqq \varphi_{0}(x) \leqq \mu$. The convergence is uniform; i.e., $\left\|\varphi_{n}-\varphi\right\|=\sup _{-1 \leqq x \leqq 1}\left|\varphi_{n}(x)-\varphi(x)\right| \rightarrow 0$. The variation of $\varphi$ with $x$ is bounded as follows:

$$
\left|\varphi\left(x_{1}\right)-\varphi\left(x_{2}\right)\right| \leqq M\left[\left|\frac{x_{1}-x_{2}}{1-x_{2}^{2}}\right|^{\frac{1}{2}}+\left|g\left(x_{1}\right)-g\left(x_{2}\right)\right|\right], \quad x_{2}^{2} \geqq x_{1}^{2}, \quad M>0
$$


According to an argument of Ref. [3] (at the end of Section 2), there is no solution at all with $\varphi(x)<0$ at any $x$, provided $\sin \mu<1$. Since it is obvious that $\varphi(x) \leqq \mu$ if $\varphi$ is a solution, it follows that the solution of Theorem 1 is the only continuous solution for which $-\pi / 2 \leqq \varphi(x) \leqq \pi / 2$, provided $g(x)$ is continuous and $\sin \mu<\sin \mu_{0}$.

In the Appendix we generalize Theorem 1 by using a strictly positive lower bound of the integral in (2.9). In the generalization, a solution is obtained for a bigger range of $\sin \mu$.

Martin also showed [following his Eq. (32)] that there cannot be two solutions in $T$ if

$$
\frac{2^{\frac{1}{2}} \sin ^{2} \mu}{\left(1-\sin ^{2} \mu \cos ^{2} \mu\right)^{\frac{1}{2}}}<1, \quad \text { i.e., } \quad \sin \mu<\sin \mu_{1} \cong 0.79 .
$$

He did not show that the mapping $A$ is a contraction when (2.17) holds, and so he could not be sure that the sequence $(2.12)$ would converge. We will now prove, however, that the operator is indeed contractive, but with a topology different from that provided by the norm (2.7).

We make use of Schwarz's inequality in the manner of Martin. The operator $B=\sin A$ will be written as

$$
B_{12}(\varphi)=\int \frac{d \Omega_{3}}{4 \pi} \frac{g_{13} g_{32}}{g_{12}} \cos \left[\varphi_{13}-\varphi_{32}\right],
$$

where $\varphi_{12}=\varphi\left(x_{12}\right)$, etc., $x_{12}$ being the cosine of the angle between the initial and final directions of the particle. Schwarz's inequality yields

$$
\begin{aligned}
& \qquad B_{12}^{2} \leqq \int \frac{d \Omega_{3}}{4 \pi} \frac{g_{13} g_{32}}{g_{12}} \int \frac{d \Omega_{3}}{4 \pi} \frac{g_{13} g_{32}}{g_{12}} \cos ^{2}\left[\varphi_{13}-\varphi_{32}\right] \\
& g_{12}^{2}\left(B_{\varphi} h\right)_{12}^{2} \leqq \int \frac{d \Omega_{3}}{4 \pi} g_{13} g_{32}\left[h_{13}-h_{32}\right]^{2} \int \frac{d \Omega_{3}}{4 \pi} g_{13} g_{32} \sin ^{2}\left[\varphi_{13}-\varphi_{32}\right] \\
& \text { After defining } \\
& w_{12}=\int \frac{d \Omega_{3}}{4 \pi} \frac{g_{13} g_{32}}{g_{12}}
\end{aligned}
$$

we combine (2.19) and (2.20) to obtain

$$
g_{12}\left(B_{\varphi} h\right)_{12}^{2} \leqq \int \frac{d \Omega_{3}}{4 \pi} g_{13} g_{32}\left[h_{13}-h_{32}\right]^{2}\left[w_{12}-\frac{B_{12}^{2}}{w_{12}}\right] \text {. }
$$

Recalling that $A_{\varphi}=\left(1-B^{2}\right)^{-\frac{1}{2}} B_{\varphi}$, we see that $\left(A_{\varphi} h\right)^{2}$ is majorized by maximizing

$$
\chi=\frac{w_{12}-B_{12}^{2} / w_{12}}{1-B_{12}}
$$

with respect to $B_{12}^{2}$. The quantity $(2.23)$ is monotonic-decreasing as a function of $B_{12}^{2}$. If $\varphi \in T$, then

$$
\cos \mu w_{12} \leqq B_{12} \leqq w_{12} \leqq \sin \mu
$$


from which it follows that

$$
\chi \leqq \frac{w_{12}\left(1-\cos ^{2} \mu\right)}{1-w_{12}^{2} \cos ^{2} \mu} \leqq \frac{\sin ^{3} \mu}{1-\sin ^{2} \mu \cos ^{2} \mu} \equiv M(\mu) .
$$

From inequality (2.22), one is led naturally to consider a Banach space $U$ consisting of all functions $\varphi$ for which the following quantity, the norm in $U$, exists:

$$
\begin{aligned}
\|\varphi\| & =\left[\int \frac{d \Omega_{1}}{4 \pi} g_{12} \varphi_{12}^{2}\right]^{\frac{1}{2}} \\
& =\left[\frac{1}{2} \int_{-1}^{1} d x g(x) \varphi^{2}(x)\right]^{\frac{1}{2}} .
\end{aligned}
$$

(The integrals here are defined in the Lebesgue sense.) We apply the contraction mapping principle in a complete metric space $V \subset U$, where

$$
V=\{\varphi: \varphi \in U, 0 \leqq \varphi(x) \leqq \mu\} .
$$

The operator $A$ of Eq. (2.6) takes $V$ into itself. Furthermore, $A$ is contractive in its action on $V$ under condition (2.17), as one sees by the following calculations. Since (2.22) and (2.25) hold if $\varphi \in V$, we have for all $h \in U$ and all $\varphi \in V$ hat

$$
g_{12}\left(A_{\varphi} h\right)_{12}^{2} \leqq M(\mu) \cdot \int \frac{d \Omega_{3}}{4 \pi} g_{13} g_{32}\left[h_{13}-h_{32}\right]^{2} .
$$

Now we integrate (2.29) over all directions of both particle 1 and particle 2. After one of the integrations, the left hand side is independent of the other direction. Thus,

$$
\begin{aligned}
& \int \frac{d \Omega_{2}}{4 \pi} \int \frac{d \Omega_{1}}{4 \pi} g_{12}\left(A_{\varphi} h\right)_{12}^{2}=\int \frac{d \Omega_{1}}{4 \pi} g_{12}\left(A_{\varphi} h\right)_{12}^{2} \\
& =\left\|A_{\varphi} h\right\|^{2} \leqq M(\mu)\left[\int \frac{d \Omega_{3}}{4 \pi} \int \frac{d \Omega_{1}}{4 \pi} g_{13} h_{13}^{2} \int \frac{d \Omega_{2}}{4 \pi} g_{32}\right. \\
& \quad+\int \frac{d \Omega_{3}}{4 \pi} \int \frac{d \Omega_{2}}{4 \pi} g_{32} h_{32}^{2} \int \frac{d \Omega_{1}}{4 \pi} g_{13} \\
& \left.\quad-2 \int \frac{d \Omega_{3}}{4 \pi} \int \frac{d \Omega_{2}}{4 \pi} g_{32} h_{32} \int \frac{d \Omega_{1}}{4 \pi} g_{13} h_{13}\right] \\
& =2 M(\mu)\left[\int \frac{d \Omega_{1}}{4 \pi} g_{13} h_{13}^{2} \int \frac{d \Omega_{2}}{4 \pi} g_{32}-\left(\int \frac{d \Omega_{1}}{4 \pi} g_{13} h_{13}\right)^{2}\right] \\
& \leqq \\
& \quad 2 M(\mu) \int \frac{d \Omega_{2}}{4 \pi} g_{32} \cdot\|h\|^{2} .
\end{aligned}
$$


Hence, a sufficient condition that $A$ be contractive is

$$
\left[\frac{2 \sin ^{3} \mu}{1-\sin ^{2} \mu \cos ^{2} \mu} \int \frac{d \Omega_{2}}{4 \pi} g_{32}\right]^{\frac{1}{2}}<1,
$$

since in this case $\left\|A_{\varphi}\right\|<1$, by (2.30). We can find a stronger sufficient condition, which implies (2.31), by integrating the inequality

$$
\int \frac{d \Omega_{3}}{4 \pi} g_{13} g_{32} \leqq g_{12} \sin \mu \text {. }
$$

We integrate over both the 1 and 3 directions and cancel a factor to obtain

$$
\int \frac{d \Omega_{2}}{4 \pi} g_{32} \leqq \sin \mu \text {. }
$$

By putting together (2.33) and (2.31), we then have condition (2.17).

It follows now from the contraction mapping principle that, if $\mu<\mu_{1}$, there is a unique solution of (2.6) in the set $V$, and that this solution is the limit with respect to the topology of the norm (2.26) of the iterative sequence $\left\{\varphi_{n}\right\}$ defined in (2.12). We can show that this solution is actually a continuous function on $[-1,1]$, as well as being merely bounded and square-integrable. The analysis of Section $\mathrm{V}$ shows that if $\varphi \in V$, then $A(x ; \varphi)$ enjoys the following property of continuity:

$$
\begin{gathered}
\left|A\left(x_{1} ; \varphi\right)-A\left(x_{2} ; \varphi\right)\right| \leqq M\left[\left|\frac{x_{1}-x_{2}}{1-x_{2}^{2}}\right|^{\alpha}+\left|g\left(x_{1}\right)-g\left(x_{2}\right)\right|\right], \\
x_{2}^{2} \geqq x_{1}^{2}, \quad M>0, \quad 0<\alpha<\frac{1}{2} .
\end{gathered}
$$

Hence, the solution of the equation in $V$ is bounded and continuous on the open interval $(-1,1)$. It is then easy to see that $A$ is continuous on the closed interval $[-1,1]$, if we return to the equation in the form (2.2). With $\Phi(x)=4 \pi g(x) B(x ; \varphi)$, Eq. (2.2) yields

$$
\begin{aligned}
\left|\Phi\left(x_{1}\right)-\Phi\left(x_{2}\right)\right| & \leqq \int_{-1}^{1} d y \int_{0}^{2 \pi} d w g(y)\left[g\left(z_{1}\right)\left|\varphi\left(z_{1}\right)-\varphi\left(z_{2}\right)\right|+\left|g\left(z_{1}\right)-g\left(z_{2}\right)\right|\right] \\
& =I_{1}+I_{2} .
\end{aligned}
$$

Since $g(x)$ is continuous on $[-1,1]$ it is uniformly continuous on $[-1,1]$. Also,

$$
\left|z_{1}-z_{2}\right| \leqq M\left|x_{1}-x_{2}\right|^{\frac{1}{2}},
$$

where $M$ is independent of $y, z, x_{1}$ and $x_{2}$. It follows that $I_{2}<\varepsilon$ for $\left|x_{1}-x_{2}\right|$ sufficiently small. To apply a similar argument to $I_{1}$, we note that $\varphi$ is bounded, so that the integral over a sufficiently small neighbour- 
hood of a point in the $y-w$ plane at which $z_{i}^{2}=1$ can be made less than $\varepsilon$, independently of $z_{i}$. The integral $I_{1}$, after deletion of such neighbourhoods, may be treated in the same way as $I_{2}$, since the integrand then is uniformly continuous. It follows that $\Phi(x)$, and hence $A(x ; \varphi)$, is continuous on $[-1,1]$.

Every iterate $\varphi_{n}$, as well as the limit $\varphi$, has the property (2.34). This allows one to show that, for $x^{2} \neq 1, \varphi_{n}(x)$ converges point-wise to $\varphi(x)$ (and not merely in the mean-square sense). We assume the contrary, that $\left|\varphi_{n}(x)-\varphi(x)\right|, x^{2} \neq 1$, does not tend to zero as $n$ goes to infinity. Then there must be an infinite set $S$ of integers, such that

$$
\left|\varphi_{n}(x)-\varphi(x)\right|>2 \alpha,
$$

for all $n \in S$. Since both $\varphi_{n}$ and $\varphi$ have the continuity property (2.34), we know that there is a $\delta>0$ such that for all $n$

$$
\begin{aligned}
\left|\varphi_{n}(x)-\varphi(x)-\varphi_{n}\left(x^{\prime}\right)+\varphi\left(x^{\prime}\right)\right| & <\alpha, \\
\left|x-x^{\prime}\right| & <\delta .
\end{aligned}
$$

We take $\delta$ so small that $x^{\prime 2}<1$ when $\left|x-x^{\prime}\right|<\delta$. From (2.37) and (2.38), it then follows that

$$
\left|\varphi_{n}\left(x^{\prime}\right)-\varphi\left(x^{\prime}\right)\right|>\alpha, \quad\left|x-x^{\prime}\right|<\delta,
$$

for all $n \in S$. Hence

$$
\frac{1}{2} \int_{-1}^{1} d x^{\prime} g\left(x^{\prime}\right)\left[\varphi_{n}\left(x^{\prime}\right)-\varphi\left(x^{\prime}\right)\right]^{2}>\delta \alpha^{2} \sup g
$$

for all $n \in S$. But this is impossible, since the integral on the left is $\left\|\varphi_{n}-\varphi\right\|^{2}$, which tends to zero as $n$ increases. We have now proved

Theorem 2. If $g(x)$ is continuous on $[-1,1]$ and $\sin \mu<\sin \mu_{1} \cong 0.79$, there is a unique solution $\varphi$ such that $0 \leqq \varphi \leqq \mu$ and such that the following Lebesgue integral, the squared norm of $\varphi$, exists:

$$
\|\varphi\|^{2}=\frac{1}{2} \int_{-1}^{1} d x g(x) \varphi^{2}(x) .
$$

This solution is the limit of the sequence $\left\{\varphi_{n}\right\}$, where $\varphi_{n+1}=A\left(\varphi_{n}\right)$, and $\varphi_{0}$ is any function such that $\left\|\varphi_{0}\right\|$ exists and $0 \leqq \varphi_{0} \leqq \mu$. The convergence is in the mean square sense $\left(\left\|\varphi-\varphi_{n}\right\| \rightarrow 0\right)$, and also point-wise except at the end-points (i.e. for each $x \neq \pm 1,\left|\varphi_{n}(x)-\varphi(x)\right| \rightarrow 0$ ). The solution $\varphi$ is continuous on $[-1,1]$ and has the property

$$
\left|\varphi\left(x_{1}\right)-\varphi\left(x_{2}\right)\right| \leqq M\left[\left|\frac{x_{1}-x_{2}}{1-x_{2}^{2}}\right|^{\alpha}+\left|g\left(x_{1}\right)-g\left(x_{2}\right)\right|\right], \quad x_{2}^{2} \geqq x_{1}^{2},
$$


where $M>0$ and $0<\alpha<\frac{1}{2}$. The conclusions are all the same if the condition $\sin \mu<\sin \mu_{1}$ is replaced by the weaker inequality (2.31).

As in the remark following Theorem 1, we can assert that the solution of Theorem 2 is actually unique in a bigger set. If $g(x) \in C[-1,1]$ and $\mu<\mu_{1}$, then there is a unique solution in the set of all functions $\varphi$ such that $\|\varphi\|$ exists and $-\pi / 2 \leqq \varphi(x) \leqq \pi / 2$.

\section{Remarks on the Application of Schauder's Theorem}

One attempts to apply Schauder's theorem [7] in the following form: If a continuous operator $A$ maps a closed convex subset $F$ of a Banach space $E$ into a compact subset of $F$, then $\varphi=A(\varphi)$ has at least one solution in $F$. (It is understood that the metric is provided by the norm in $E: d(\varphi, \psi)=\|\varphi-\psi\|$.

Newton [2] identified the Banach space $E$ with the space $S$ defined in the previous section; i.e. the space of real, continuous functions on $[-1,1]$ with the uniform norm (2.7). Newton's argument is based on the assumption that $A(T)$ is compact, where $T$ is defined in Eq. $(2.8)^{1}$. We shall now show, by means of a counter example, that $A(T)$ is in fact not compact.

The idea of our proof is to display a sequence $\left\{\varphi_{n}\right\}$ of functions, $\varphi_{n} \in T$, in which $\varphi_{n}$ oscillates with increasing frequency as $n$ tends to infinity. By means of simple estimates, we find that the functions $A\left(\varphi_{n}\right)$, $n=1,2, \ldots$ form a non-equicontinuous set. By the necessity clause of the Ascoli-Arzelà theorem [6], it follows that the $A\left(\varphi_{n}\right)$ form a noncompact set.

We first define a set of piecewise-continuous functions $\psi_{n}(x)$, which approximate the $\varphi_{n}(x)$ (in a sense to be specified later):

$$
\begin{aligned}
\psi_{n}(x)=\left\{\begin{array}{lr}
\varepsilon, & k<n x<k+1, \\
0, & k+1<n x<k+2,
\end{array}\right. \\
k=-n, \quad-n+2, \ldots, n-2, \\
0<\varepsilon<\mu<\pi / 2 .
\end{aligned}
$$

To make the example as simple as possible, we take an amplitude with constant differential cross-section: $g(x)=g \leqq \sin \mu$. We first estimate the unitarity integral for the approximating amplitude

$$
g e^{i \psi_{n}(x)} .
$$

1 Prof. Newton has corrected his proof by using a space of Hölder-continuous functions (private communication). This technique requires $g(x)$ to be Hölder-continuous, with a sufficiently small Hölder coefficient. Our proofs of Sections 4 and 5 require only ordinary continuity of $g(x)$. 
With the variables of Eq. (2.3), the integral is

with

$$
\begin{aligned}
B\left(x ; \psi_{n}\right) & =\frac{g}{4 \pi} \int_{-1}^{1} d y \int_{0}^{2 \pi} d w \cos \left[\psi_{n}(y)-\psi_{n}(z)\right] \\
& =\frac{g}{4 \pi}\left(\Omega_{+}+\cos \varepsilon \Omega_{-}\right),
\end{aligned}
$$

$$
z=x y+\left[\left(1-x^{2}\right)\left(1-y^{2}\right)\right]^{\frac{1}{2}} \cos w .
$$

Here $\Omega_{+}$is the solid angle in which $\psi_{n}(y)=\psi_{n}(z)$, and $\Omega_{-}$that in which $\psi_{n}(y)-\psi_{n}(z)= \pm \varepsilon$. We first prove the following Lemma: There exists a solid angle $w>0$, independent of $x$, such that $\Omega_{-} \geqq w$ when $n>n_{0}(x)$, for any $x \neq \pm 1$.

Let $\chi(x, y, w)$ be the characteristic function of the set on which $\psi_{n}(y)-\psi_{n}(z)= \pm \varepsilon$; that is, $\chi=1$ on this set and $\chi=0$ elsewhere. Then

$$
\begin{aligned}
\Omega_{-} & =\int_{-1}^{1} d y \int_{0}^{2 \pi} d w \chi(x, y, w) \\
& \geqq \int_{-\frac{1}{2}}^{\frac{1}{2}} d y \int_{0}^{2 \pi} d w \chi(x, y, w) \\
& =2 \int_{-\frac{1}{2}}^{\frac{1}{2}} d y \int_{-1}^{1} \frac{d v}{\left(1-v^{2}\right)^{\frac{1}{2}}} \chi(x, y, w(v)),
\end{aligned}
$$

where $v=\cos w$. For fixed $y$, the variation of $z$ as $v$ varies from -1 to 1 is

$$
\Delta z=2\left[\left(1-x^{2}\right)\left(1-y^{2}\right)\right]^{\frac{1}{2}} \geqq 2\left[\left(1-x^{2}\right) \frac{3}{4}\right]^{\frac{1}{2}} .
$$

Since we have $x^{2} \neq 1$, we may choose $n_{0}(x)$ such that

$$
n_{0}(x) \Delta z \geqq 2 n_{0}(x)\left[\left(1-x^{2}\right) \frac{3}{4}\right]^{\frac{1}{2}} \geqq 2 .
$$

Then for any $y$ and $n \geqq n, \psi_{n}(z)$ is equal to $\varepsilon$ over at least one third of the interval $-1 \leqq v \leqq 1$, and equal to zero over at least one third of the same interval. Hence $\left|\psi_{n}(y)-\psi_{n}(z)\right|=\varepsilon$ over at least a third of the interval. Since $\left(1-v^{2}\right)^{-\frac{1}{2}}$ is smallest near $v=0$, we have the desired lower bound on $\Omega_{-}$:

$$
\Omega_{-} \geqq 2 \int_{-\frac{1}{2}}^{\frac{1}{2}} d y \int_{-\frac{1}{3}}^{\frac{1}{3}} \frac{d v}{\left(1-v^{2}\right)^{\frac{1}{2}}}=w>0 .
$$

Having proved the lemma, we now prove that the functions $B\left(x ; \psi_{n}\right)$ form a non-equicontinuous set.

From (3.3) and the relation $\Omega_{+}+\Omega_{-}=4 \pi$ we have

$$
\begin{aligned}
B\left(x ; \psi_{n}\right) & =\frac{g}{4 \pi}\left[4 \pi-(1-\cos \varepsilon) \Omega_{-}\right] \\
& \leqq g-\frac{g}{4 \pi}(1-\cos \varepsilon) w \equiv g_{0}<g
\end{aligned}
$$


Eq. (3.9) holds for any $x \neq \pm 1$, with $g_{0}$ independent of $x$, provided $n>n_{0}(x)$. On the other hand, $B\left(1 ; \psi_{n}\right)=g$, so that for sufficiently large $n$, $B\left(x ; \psi_{n}\right)$ becomes arbitrarily sharply peaked at $x=1$. Equicontinuity of the functions $B\left(x ; \psi_{n}\right)$ would require that for any $\varepsilon>0$ there exists a $\delta$ independent of $n$ such that

$$
\left|B\left(1 ; \psi_{n}\right)-B\left(x ; \psi_{n}\right)\right|<\varepsilon
$$

when $|1-x|<\delta$. However, we see from (3.9) that the $B\left(x ; \psi_{n}\right)$ are not equicontinuous, since for any $\delta>0$ and $|1-x|<\delta$ we can choose an $n$ so large that

$$
\left|B\left(1 ; \psi_{n}\right)-B\left(x ; \psi_{n}\right)\right| \geqq g-g_{0} .
$$

The continuous functions $\varphi_{n}$, which approximate $\psi_{n}$, may be chosen as follows (see Fig. 1):

$$
\varphi_{n}(x)= \begin{cases}\varepsilon & k+\eta \leqq n x \leqq k+1-\eta \\ \frac{-\varepsilon}{2 \eta}(n x+k+1+\eta) & \text { and }-n \leqq n x \leqq-n+\delta, \\ 0 & k+1-\eta \leqq n x \leqq k+1+\eta, \\ \frac{\varepsilon}{2 \eta}(n x-k-2+\eta) & k+2-\eta \leqq n x \leqq k+2+\eta, \\ & k=-n,-n+2, \ldots, n-2 .\end{cases}
$$

Now we can show that for any $\delta>0$ and $|1-x|<\delta$, there is an $n$ and an $\eta$ such that

$$
\left|B\left(1 ; \varphi_{n}\right)-B\left(x ; \varphi_{n}\right)\right| \geqq g-g_{0}-K \eta,
$$

$K>0$ being independent of $x$ and $n$. Thus, if $\eta$ is chosen so that $K \eta<g-g_{0}$, the $B\left(x ; \varphi_{n}\right)$ are not equicontinuous. To establish (3.13), note that

$$
\begin{aligned}
& \left|B\left(1 ; \varphi_{n}\right)-B\left(x ; \varphi_{n}\right)\right| \\
& \quad=\left|B\left(1 ; \varphi_{n}\right)-B\left(1 ; \psi_{n}\right)-B\left(x ; \varphi_{n}\right)+B\left(x ; \psi_{n}\right)+B\left(1 ; \psi_{n}\right)-B\left(x ; \psi_{n}\right)\right| \\
& \quad \geqq|| B\left(1 ; \psi_{n}\right)-B\left(x ; \psi_{n}\right)|-| B\left(x ; \psi_{n}\right)-B\left(x ; \varphi_{n}\right)|| \\
& \quad \geqq g-g_{0}-\left|B\left(x ; \psi_{n}\right)-B\left(x ; \varphi_{n}\right)\right|,
\end{aligned}
$$

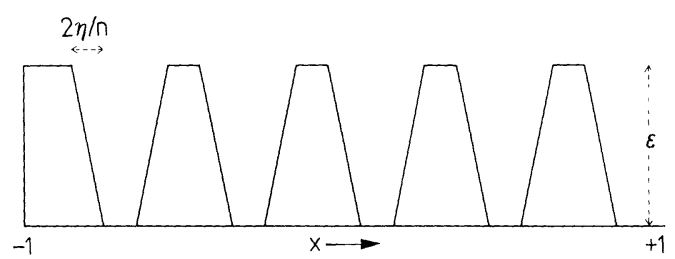

Fig. 1. The continuous function $\varphi_{n}(\mathrm{x})$ of Eq. (3.12), with $n=5$ 
provided that the last quantity on the right can be made less than $g-g_{0}$. But now note that $\left|\varphi_{n}(y)-\psi_{n}(y)\right|$ is zero, except in $2 n-1$ intervals of length $2 \eta / n$ each, where it is bounded above by $\varepsilon / 2$. Consequently,

$$
\begin{aligned}
\mid B\left(x ; \psi_{n}\right) & -B\left(x ; \varphi_{n}\right) \mid \\
& \leqq g \int_{-1}^{1} d y \int_{0}^{2 \pi} d w\left|\cos \left(\psi_{n}(x)-\psi_{n}(z)\right)-\cos \left(\varphi_{n}(x)-\varphi_{n}(z)\right)\right| \\
& \leqq g \int_{-1}^{1} d y \int_{0}^{2 \pi} d w\left|\psi_{n}(x)-\varphi_{n}(x)-\psi_{n}(z)+\varphi_{n}(z)\right| \\
& \leqq 4 \pi g \frac{(2 n-1)}{n} \eta \varepsilon<K \eta .
\end{aligned}
$$

We have now established that the $B\left(x ; \varphi_{n}\right)$ are not equicontinuous, and to complete the proof need note only that the same is true of the $A\left(x ; \varphi_{n}\right)=\sin ^{-1} B\left(x ; \varphi_{n}\right)$. This follows from the mean value theorem:

$$
\begin{aligned}
\left|A\left(1 ; \varphi_{n}\right)-A\left(x ; \varphi_{n}\right)\right| & =\left(1-B^{2}\left(\xi ; \varphi_{n}\right)\right)^{-\frac{1}{2}}\left|B\left(1 ; \varphi_{n}\right)-B\left(x ; \varphi_{n}\right)\right| \\
& \geqq\left|B\left(1 ; \varphi_{n}\right)-B\left(x ; \varphi_{n}\right)\right|, \quad x<\xi<1 .
\end{aligned}
$$

Martin recognised the difficulty of proving the compactness of $A(T)$. In order to overcome this difficulty, he replaced $T$ by $\hat{T}$, defined to be the closure of the convex hull of the set $A(T)$. Martin proved that the set $A(\hat{T})$ is compact, and was able to apply Schauder's theorem. However, in the course of the demonstration of compactness, he employed a partial-wave series, the convergence of which was not discussed. In the following section, we show that the convergence question can be treated satisfactorily, and we thus complete the Schauder proof (which we do in a way slightly different from that of Martin).

\section{Schauder Fixed-Point Proof with Partial Waves}

We shall now describe an existence proof using Schauder's theorem and a partial-wave development. The first step is to state the unitarity condition in terms of partial waves. In scattering theory, this is normally a trivial step, since one is dealing with analytic functions, and so the Legendre series converges absolutely and uniformly. In the present instance, in which only continuity of $f(x)$ is available, we cannot assert the point-wise convergence of the series

$$
\sum_{l=0}^{\infty}(2 l+1) f_{l} P_{l}(x)
$$

where the $f_{l}$ are the Legendre coefficients of $f(x)$, namely

$$
f_{l}=\frac{1}{2} \int_{-1}^{1} d x P_{l}(x) f(x)
$$


(The series (4.1) does converge in the mean to $f(x)$, but this is not sufficient in itself to justify Eq. 8 of Ref. [2]).

Let us first note that since the function $f(x)$ is continuous on the closed interval $[-1,1]$, it may be uniformly approximated by polynomials on this interval. Specifically, the Weierstrass approximation theorem guarantees that for any $\varepsilon>0$ there exists a polynomial $S_{L}(x)$ of degree $L$ such that

$$
\left|f(x)-S_{L}(x)\right|<\varepsilon, \quad-1 \leqq x \leqq 1 .
$$

We may express $S_{L}$ in terms of Legendre polynomials:

$$
S_{L}(x)=\sum_{l=0}^{L} b_{l}^{L} P_{l}(x)
$$

Note that the (flexible) coefficients $b_{l}^{L}$ depend on $L$ as well as on $l$. We define an auxiliary function $I(x)$ as

$$
I(x)=\int_{-1}^{1} d y \int_{0}^{2 \pi} d w\left[f(y)-S_{L}(y)\right]\left[f^{*}(z)-S_{L}^{*}(z)\right],
$$

where the asterisk denotes complex conjugation and

$$
z=x y+\left[\left(1-x^{2}\right)\left(1-y^{2}\right)\right]^{\frac{1}{2}} \cos w .
$$

The bound (4.3) yields

$$
|I(x)| \leqq 4 \pi \varepsilon^{2}
$$

We may use the identity

to obtain

$$
\frac{1}{2 \pi} \int_{0}^{2 \pi} d w P_{l}(z)=P_{l}(x) P_{l}(y)
$$

$$
I(x)=\int_{-1}^{1} d y \int_{0}^{2 \pi} d w f(y) f^{*}(z)-4 \pi \sum_{l=0}^{L}(2 l+1)\left\{\left|f_{l}\right|^{2}-\left|b_{l}^{L}-f_{l}\right|^{2}\right\} P_{l}(x) .
$$

Thus we have

$$
\frac{1}{4 \pi} \int_{-1}^{1} d y \int_{0}^{2 \pi} d w f(y) f(z)=\lim _{L \rightarrow \infty} \sum_{l=0}^{L}(2 l+1)\left\{\left|f_{l}\right|^{2}-\left|b_{l}^{L}-f_{l}\right|^{2}\right\} P_{l}(x),
$$

where the limit $L \rightarrow \infty$ is approached uniformly in $x$. By the usual completeness and orthogonality of the Legendre polynomials, we have the Parseval equation, which holds for any square-integrable function $f$ :

$$
\lim _{L \rightarrow \infty} \sum_{l=0}^{L}(2 l+1)\left|f_{l}\right|^{2}=\frac{1}{2} \int_{-1}^{1} d y|f(y)|^{2}<\infty .
$$


On the other hand, by putting $x=1$ in (4.9) we obtain

$$
\lim _{L \rightarrow \infty} \sum_{l=0}^{L}(2 l+1)\left\{\left|f_{l}\right|^{2}-\left|b_{l}^{L}-f_{l}\right|^{2}\right\}=\frac{1}{2} \int_{-1}^{1} d y|f(y)|^{2} .
$$

Hence,

$$
\lim _{L \rightarrow \infty} \sum_{l=0}^{L}(2 l+1)\left|b_{l}^{L}-f_{l}\right|^{2}=0,
$$

Since $\left|P_{l}(x)\right| \leqq 1$, we have also that

and

$$
\lim _{L \rightarrow \infty} \sum_{l=0}^{L}(2 l+1)\left|f_{l}\right|^{2} P_{l}(x)<\infty
$$

$$
\lim _{L \rightarrow \infty} \sum_{l=0}^{L}(2 l+1)\left|b_{l}^{L}-f_{l}\right|^{2} P_{l}(x)=0 .
$$

It then follows from (4.9) that

$$
\frac{1}{4 \pi} \int_{-1}^{1} d y \int_{0}^{2 \pi} d w f(y) f^{*}(z)=\sum_{l=0}^{\infty}(2 l+1)\left|f_{l}\right|^{2} P_{l}(x),
$$

where the series on the right converges absolutely and uniformly with respect to $x$ in $[-1,1]$. This completes the justification of Eq. 8 of Ref. [3].

Our fixed point problem may now be written in the form

where

$$
\begin{aligned}
\varphi(x) & =A(x ; \varphi)=\sin ^{-1} B(x ; \varphi), \\
\bar{B}(x ; \varphi) & =g(x) B(x ; \varphi)=\sum_{l=0}^{\infty}(2 l+1)\left|f_{l}[\varphi]\right|^{2} P_{l}(x),
\end{aligned}
$$

$$
\mathrm{f}_{l}[\varphi]=\frac{1}{2} \int_{-1}^{1} \mathrm{dx} P_{l}(\mathrm{x}) \mathrm{g}(\mathrm{x}) \mathrm{e}^{i \varphi(x)} .
$$

We now investigate the continuity properties of functions in the set $A(T)$, where $T$ is defined in Eq. (2.8). When $\varphi \in T$, we have

$$
\left|\bar{B}\left(x_{1} ; \varphi\right)-\bar{B}\left(x_{2} ; \varphi\right)\right| \leqq \sum_{l=0}^{\infty}(2 l+1)\left|f_{l}[\varphi]\right|^{2}\left|P_{l}\left(x_{1}\right)-P_{l}\left(x_{2}\right)\right|,
$$

where the series converges, by comparison with (4.11). We can now invoke Martin's bound on $\left|P_{l}\left(x_{1}\right)-P_{l}\left(x_{2}\right)\right|$ (Eq. 10 of Ref. [3]) to obtain

$$
\begin{aligned}
\left|\bar{B}\left(x_{1} ; \varphi\right)-\bar{B}\left(x_{2} ; \varphi\right)\right| & \leqq M \frac{\left|\cos ^{-1} x_{1}-\cos ^{-1} x_{2}\right|^{\frac{1}{2}}}{\left|\left(1-x_{1}^{2}\right)\left(1-x_{2}^{2}\right)\right|^{\frac{1}{8}}} \int_{-1}^{1} d y|g(y)|^{2} \\
& \leqq M \frac{\left|x_{1}-x_{2}\right|^{\frac{1}{2}}}{\left|1-x_{2}^{2}\right|^{\frac{1}{2}}},
\end{aligned}
$$


where we have chosen $x_{2}^{2} \geqq x_{1}^{2}$, and we have used the mean value theorem for $\cos ^{-1} x$. Hence by (4.18) and (4.21)

$$
\begin{aligned}
& \left|B\left(x_{1} ; \varphi\right)-B\left(x_{2} ; \varphi\right)\right| \leqq\left|\frac{g\left(x_{1}\right)-g\left(x_{2}\right)}{g\left(x_{1}\right) g\left(x_{2}\right)}\right| \bar{B}\left(x_{1} ; \varphi\right) \\
& \quad+\frac{1}{g\left(x_{2}\right)}\left|\bar{B}\left(x_{1} ; \varphi\right)-\bar{B}\left(x_{2} ; \varphi\right)\right| \leqq M \frac{\left|x_{1}-x_{2}\right|^{\frac{1}{2}}}{\left|1-x_{2}^{2}\right|^{\frac{1}{2}}}+M\left|g\left(x_{1}\right)-g\left(x_{2}\right)\right|,
\end{aligned}
$$

where the constant $M$ is independent of $\varphi$. Finally, we use the mean value theorem for $\sin ^{-1} B$, and the fact that $|B(\varphi)| \leqq \sin \mu<1, \varphi \in T$, to obtain $\left|A\left(x_{1} ; \varphi\right)-A\left(x_{2} ; \varphi\right)\right| \leqq M \frac{\left|x_{1}-x_{2}\right|^{\frac{1}{2}}}{\left|1-x_{2}^{2}\right|^{\frac{1}{2}}}+M\left|g\left(x_{1}\right)-g\left(x_{2}\right)\right|, \quad x_{2}^{2} \geqq x_{1}^{2}$.

The factor $\left|1-x_{2}^{2}\right|^{-\frac{1}{2}}$ in (4.23) is a manifestation of the lack of equicontinuity of the set of functions $A(x ; \varphi), \varphi \in T$, that we made explicit in Section 3. To deal with this factor, we rewrite the unitarity equation as follows:

$$
\begin{aligned}
\psi(x) & =C(x ; \psi), \\
C(x ; \psi) & =\left(1-x^{2}\right)^{\frac{1}{2}} A(x ; \varphi),
\end{aligned}
$$

where in (4.25) we put

$$
\varphi(x)=\left(1-x^{2}\right)^{-\frac{1}{2}} \psi(x) .
$$

The following set, $V$, is bounded, closed, and convex with respect to the norm topology of the Banach space $S$ defined in (2.7):

$$
V=\left\{\psi: \psi \in S, 0 \leqq \psi(x) \leqq\left(1-x^{2}\right)^{\frac{1}{2}} \mu, 0 \leqq x \leqq 1\right\}
$$

The operator $C$ maps $V$ into itself, provided $\sin \mu<1$. Also, $C$ is continuous on $V$, according to the following argument:

$$
\begin{aligned}
& \left|A\left(x ; \varphi_{1}\right)-A\left(x ; \varphi_{2}\right)\right| \\
& \quad \leqq \frac{1}{4 \pi} \int_{-1}^{1} d y \int_{0}^{2 \pi} d w g(y) g(z)\left|\cos \left[\varphi_{1}(y)-\varphi_{1}(z)\right]-\cos \left[\varphi_{2}(y)-\varphi_{2}(z)\right]\right| \\
& \quad \leqq \frac{1}{4 \pi} \int_{-1}^{1} d y \int_{0}^{2 \pi} d w g(y) g(z)\left(\frac{\left|\psi_{1}(y)-\psi_{2}(y)\right|}{\left(1-y^{2}\right)^{\frac{1}{2}}}+\frac{\left|\psi_{1}(z)-\psi_{2}(z)\right|}{\left(1-z^{2}\right)^{\frac{1}{2}}}\right) \\
& \quad=\frac{2}{4 \pi} \int_{-1}^{1} d y \int_{0}^{2 \pi} d w g(y) g(z) \frac{\left|\psi_{1}(y)-\psi_{2}(y)\right|}{\left(1-y^{2}\right)^{\frac{1}{2}}} \\
& \quad \leqq M \sup _{-1 \leqq y}\left|\psi_{1}(y)-\psi_{2}(y)\right| .
\end{aligned}
$$


Hence $\left\|C\left(\psi_{1}\right)-C\left(\psi_{2}\right)\right\| \leqq M\left\|\psi_{1}-\psi_{2}\right\|$. Next we remark that the set $C(V)$ is compact by virtue of the Ascoli-Arzelà theorem and Eq. (4.23). That is, with $x_{2}^{2} \geqq x_{1}^{2}$ we have

$$
\begin{aligned}
& \left|C\left(x_{1} ; \psi\right)-C\left(x_{2} ; \psi\right)\right|=\mid\left(1-x_{1}^{2}\right)^{\frac{1}{2}}\left[A\left(x_{1} ; \psi\right)-A\left(x_{2} ; \psi\right)\right] \\
& \quad+A\left(x_{2} ; \psi\right)\left[\left(1-x_{1}^{2}\right)^{\frac{1}{2}}-\left(1-x_{2}^{2}\right)^{\frac{1}{2}}\right]|\leqq M| x_{1}-\left.x_{2}\right|^{\frac{1}{2}}+M\left|g\left(x_{1}\right)-g\left(x_{2}\right)\right| .
\end{aligned}
$$

Since we have assumed that $g(x)$ is continuous, the functions $C(x ; \psi)$, $\psi \in V$, are uniformly bounded and equicontinuous, so $C(V)$ is a compact subset of $V$, by the Ascoli-Arzelà theorem. It follows from Schauder's theorem that there is at least one solution of Eq. (4.24) in $V$. There is, therefore, at least one solution of $\varphi=A(\varphi)$ in $T$.

Theorem 3. If $g(x)$ is continuous, $-1 \leqq x \leqq 1$, and $\sin \mu<1$, there is at least one continuous solution $\varphi(x)$ such that $0 \leqq \varphi(x) \leqq \mu,-1 \leqq x \leqq 1$. Every such solution has the property

$$
\left|\varphi\left(x_{1}\right)-\varphi\left(x_{2}\right)\right| \leqq M\left[\left|\frac{x_{1}-x_{2}}{1-x_{2}^{2}}\right|^{\frac{1}{2}}+\left|g\left(x_{1}\right)-g\left(x_{2}\right)\right|\left[, \quad M>0, \quad x_{2}^{2} \geqq x_{1}^{2} .\right.\right.
$$

\section{Schauder Fixed-Point Proof without Partial Waves}

We now give another proof of the theorem of the last section, which is more elementary in that it avoids delicate convergence questions, and does not require a bound for the modulus of Hölder-continuity of Legendre functions.

As in the previous section, we apply Schauder's theorem to the equation in the form (4.24), considering $C$ as a mapping of $V$ into itself. The new proof differs from the previous one only in that we use a different method to obtain an inequality similar to (4.21). We work with the operator $\bar{B}=g \sin A$ in the form (2.4), and begin by noting that for any $\psi \in V$ we have

$$
\left|\bar{B}\left(x_{1} ; \varphi\right)-\bar{B}\left(x_{2} ; \varphi\right)\right| \leqq \frac{1}{2 \pi} \iint d y d z g(y) g(z)\left|\frac{\chi_{1}(y, z)}{k\left(x_{1}, y, z\right)}-\frac{\chi_{2}(y, z)}{k\left(x_{2}, y, z\right)}\right|,
$$

where

$$
k(x, y, z)=\left[1-x^{2}-y^{2}-z^{2}+2 x y z\right]^{\frac{1}{2}},
$$

and $\chi_{i}(y, z)$ is the characteristic function of the region where $k^{2}\left(x_{i}, y, z\right)>0$, $i=1,2$. It is convenient to decompose $\chi_{i}$ as

$$
\chi_{i}(y, z)=\chi(y, z)+\hat{\chi}_{i}(y, z),
$$


where $\chi=1$ when $\chi_{1}=\chi_{2}=1$, and is zero elsewhere. Then

$$
\begin{aligned}
& \left|\bar{B}\left(x_{1} ; \varphi\right)-\bar{B}\left(x_{2} ; \varphi\right)\right| \leqq \frac{1}{2 \pi} \iint d y d z g(y) g(z) \\
& \quad \cdot\left[\chi(x, z)\left|\frac{1}{k\left(x_{1}, y, z\right)}-\frac{1}{k\left(x_{2}, y, z\right)}\right|+\frac{\hat{\chi}_{1}(y, z)}{k\left(x_{1}, y, z\right)}+\frac{\hat{\chi}_{2}(y, z)}{k\left(x_{2}, y, z\right)}\right] \\
& \quad \equiv D_{1}+D_{2}+D_{3} .
\end{aligned}
$$

The following change of variable is convenient:

$$
u=2^{-\frac{1}{2}}(y-z) ; \quad v=2^{-\frac{1}{2}}(y+z) \text {. }
$$

In these variables, one sees that the curve $k^{2}=0$ is an ellipse, since

$$
k^{2}(x, y, z)=\left(1-x^{2}\right)\left[1-\frac{u^{2}}{1-x}-\frac{v^{2}}{1+x}\right] \text {. }
$$

We consider now the first term of (5.4) as $D_{1}=D_{12}+D_{21}$, where $D_{12}\left(D_{21}\right)$ is that part of the integral where $k\left(x_{1}, y, z\right)$ is greater (less) than $k\left(x_{2}, y, z\right)$. To estimate $D_{12}$, we use the inequality

$$
\left|\frac{1}{k_{1}}-\frac{1}{k_{2}}\right| \leqq \frac{1}{k_{2}^{1+2 \alpha}}\left|k_{1}^{2}-k_{2}^{2}\right|^{\alpha} \leqq 4^{\alpha} \frac{\left|x_{1}-x_{2}\right|^{\alpha}}{k_{2}^{1+2 \alpha}}, \quad 0<\alpha<\frac{1}{2} .
$$

With the variables $r_{2}, \vartheta_{2}$, where

$$
u=\left(1-x_{2}\right)^{\frac{1}{2}} r_{2} \cos \vartheta_{2}, \quad v=\left(1+x_{2}\right)^{\frac{1}{2}} r_{2} \sin \vartheta_{2},
$$

we have

$$
D_{12} \leqq M\left|\frac{x_{1}-x_{2}}{1-x_{2}^{2}}\right|^{\alpha} \int_{0}^{1} \frac{r_{2} d r_{2}}{\left(1-r_{2}^{2}\right)^{\frac{1}{2}+\alpha}} \int_{0}^{2 \pi} d \vartheta_{2},
$$

where we have majorized the integral by extending the $\theta_{2}$ integration to the entire region $[0,2 \pi]$. A similar treatment of $D_{21}\left(k_{1}\right.$ and $k_{2}$ being interchanged) yields finally

$$
\begin{gathered}
D_{1}\left(x_{1}, x_{2}\right) \leqq M\left|\frac{x_{1}-x_{2}}{1-x_{2}^{2}}\right|^{\alpha}, \\
0<\alpha<\frac{1}{2}, \quad x_{2}^{2} \geqq x_{1}^{2} .
\end{gathered}
$$

We majorize the term $D_{2}$ by replacing the integration domain by the annulus formed between the two ellipses

and

$$
\frac{u^{2}}{1-x_{1}}+\frac{v^{2}}{1+x_{1}}=1
$$

$$
\frac{u^{2}}{1-x_{1}}+\frac{v^{2}}{1+x_{1}}=\frac{1+x_{2}}{1+x_{1}}=Q^{2} \text {, }
$$




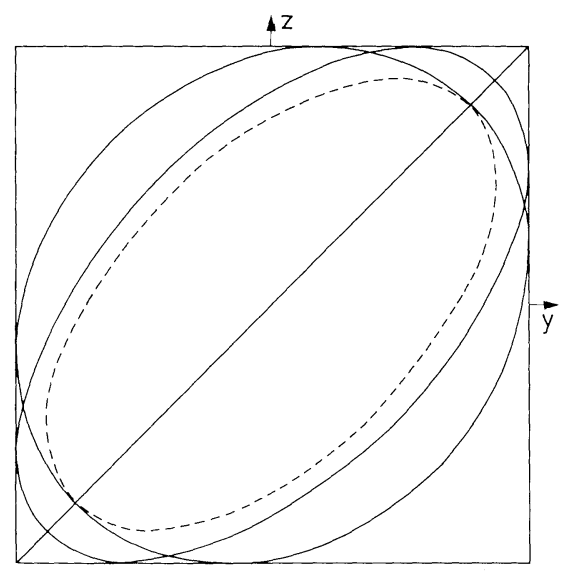

Fig. 2. The ellipses (5.11) and (5.15) (solid), and (5.12) (dotted)

where we are taking first the case $x_{2}<0$, so $-1 \leqq x_{2} \leqq x_{1} \leqq-x_{2} \leqq 1$. It may be shown that this elliptical annulus contains the support of $\hat{\chi}_{1}$ (see Fig. 2). Moreover, $k\left(x_{1}, y, z\right)$ is positive in this region. In terms of the variables $r_{1}, \vartheta_{1}$, where

$$
u=\left(1-x_{1}\right)^{\frac{1}{2}} r_{1} \cos \vartheta_{1}, \quad v=\left(1+x_{1}\right)^{\frac{1}{2}} r_{1} \sin \vartheta_{1}
$$

we have

$$
\begin{aligned}
D_{2} \leqq \frac{M}{2 \pi} \int_{\varrho}^{1} \frac{r_{1} d r_{1}}{\left(1-r_{1}^{2}\right)^{\frac{1}{2}}} \int_{0}^{2 \pi} d \vartheta_{1} & =M\left(\frac{x_{1}-x_{2}}{1+x_{1}}\right)^{\frac{1}{2}} \\
& \leqq M\left(\frac{x_{1}-x_{2}}{1+x_{1}}\right)^{\alpha}
\end{aligned}
$$

where $\alpha \leqq \frac{1}{2}$. Similarly, we majorize $D_{3}$ by integrating over the annulus between the ellipses

and

$$
\frac{u^{2}}{1-x_{2}}+\frac{v^{2}}{1+x_{2}}=1
$$

$$
\frac{u^{2}}{1-x_{2}}+\frac{v^{2}}{1+x_{2}}=\frac{1-x_{1}}{1-x_{2}} \text {. }
$$

This time we transform to $r_{2}, \vartheta_{2}$, Eq. (5.8), and obtain

$$
D_{3} \leqq M\left(\frac{x_{1}-x_{2}}{1-x_{2}}\right)^{\frac{1}{2}} \leqq M\left(\frac{x_{1}-x_{2}}{1+x_{1}}\right)^{\alpha}
$$


In much the same way we find that, when $x_{2}>0$, so $-1 \leqq-x_{2} \leqq x_{1}$ $\leqq x_{2} \leqq 1$,

$$
D_{2} \leqq M\left(\frac{x_{2}-x_{1}}{1-x_{1}}\right)^{\alpha}, \quad D_{3} \leqq M\left(\frac{x_{2}-x_{1}}{1-x_{1}}\right)^{\alpha} .
$$

Thus we certainly have, whenever $x_{2}^{2} \geqq x_{1}^{2}$,

$$
D_{2}+D_{3} \leqq M\left|\frac{x_{1}-x_{2}}{1-x_{2}^{2}}\right|^{\alpha} \text {. }
$$

We now may combine the results (5.10) and (5.19) to obtain an inequality which can be used in place of (4.21) in the proof of the previous section; namely

$$
\begin{gathered}
\left|\bar{B}\left(x_{1} ; \varphi\right)-\bar{B}\left(x_{2} ; \varphi\right)\right| \leqq M\left|\frac{x_{1}-x_{2}}{1-x_{2}^{2}}\right|^{\alpha}, \\
0<\alpha<\frac{1}{2}, \quad x_{2}^{2} \geqq x_{1}^{2} .
\end{gathered}
$$

The rest of the proof is essentially the same as in Section 4 .

\section{Inelastic Régime}

To allow for inelasticity, we add a term to the right hand side of Eq. (2.3):

$$
\begin{gathered}
\sin \varphi=B(\varphi)+I(\eta), \\
I(\eta)=\frac{1}{g(x)} \sum_{l=0}^{\infty}(2 l+1) \frac{\left(1-\eta_{l}^{2}\right)}{4} P_{l}(x), \quad 0 \leqq \eta_{l} \leqq 1 .
\end{gathered}
$$

The elasticity is denoted by $\eta_{l}$, where the partial-wave amplitude is

$$
A_{l}=\frac{\eta_{l} e^{2 i \delta_{l}}-1}{2 i} \text {. }
$$

The inelastic term $g I$ is usually assumed to be analytic in $x$ in an ellipse with foci at $x= \pm 1$, from which it follows that the series of $(6.2)$ converges absolutely and uniformly for $-1<x<1$. We need only the weaker assumption that the series converges to a continuous function of $x$.

It is possible to prove existence theorems for Eq. (6.1) by the same methods that were used in Sections 2, 4, and 5 (i.e., by the contraction mapping principle or by Schauder's theorem). The principal change concerns the condition that the set $0 \leqq \varphi \leqq \mu<\pi / 2$ be mapped into itself. That condition now takes the form

$$
\sup _{-1 \leqq x \leqq 1}\left[\iint d y d z H(x, y, z)+|I(x ; \eta)|\right] \leqq \sin \mu .
$$


Under condition (6.3), and the requirement that $g(x)$ and $I(x, \eta)$ be continuous, there is at least one continuous solution $\varphi(x)$ of $(6.1)$, such that $0 \leqq \varphi(x) \leqq \mu$, provided $\sin \mu<1$. This follows from an application of Schauder's theorem, as in Sections 4 and 5.

For the contraction mapping proof, it is convenient first to define $\sin \mu^{\prime} \leqq \sin \mu$ as follows:

$$
\sin \mu^{\prime}=\sup _{-1 \leqq x \leqq 1} \iint H(x, y, z) d y d z .
$$

By repeating the argument of Section 2, we now find that the operator will satisfy the contraction conditions if (6.3) holds and

$$
2 \sin \mu^{\prime} \tan \mu<1 \text {. }
$$

Implementation of condition (6.3) is slightly awkward, so it might be convenient to replace it by the following stronger inequality, which is an explicit restriction on $I$ :

$$
\sup _{-1 \leqq x \leqq 1}|I(x ; \eta)| \leqq \sin \mu-\sin \mu^{\prime} .
$$

If conditions (6.3) and (6.5), or (6.6) and (6.5), are satisfied, then there exists for each choice of $I$ and $g$ a unique continuous solution $\varphi$ such that $0 \leqq \varphi(x) \leqq \mu$.

For a fixed $g$, we can vary $I$ within the limits of inequality (6.6), and still have a solution. By means of the implicit function theorem, we can show that $\varphi$ depends continuously on $I$. We write

$$
F(\varphi, I)=\sin \varphi-B(\varphi)-I,
$$

and notice that $F$ has continuous Fréchet derivatives with respect to both $\varphi$ and $I$ in a neighbourhood of a point $\left(\varphi_{0}, I_{0}\right)$, where $\varphi_{0}(x)$ and $I_{0}(x)$ are any functions continuous on $[-1,1]$. Note that $-F_{I}=1$ is the unit operator. Let $\varphi_{0}$ be the unique solution obtained from the contraction mapping principle under conditions (6.3) and (6.5), with $I=I_{0}$. Then

$$
F\left(\varphi_{0}, I_{0}\right)=0,
$$

and if $F_{\varphi}^{-1}\left(\varphi_{0}, I_{0}\right)$ exists, the implicit function theorem [6] guarantees the existence of a unique function of $I$, denoted by $\varphi(x ; I)$ or $\varphi(I)$, which is continuously differentiable in some neighbourhood $\Omega$ of $I_{0}$, and such that

(i) $F(\varphi(I), I)=0, \quad I \in \Omega$,

(ii) $\varphi\left(I_{0}\right)=\varphi_{0}$,

(iii) $\frac{d \varphi}{d I}=F_{\varphi}^{-1}(\varphi(I)), \quad I \in \Omega$. 
The existence of $F_{\varphi}^{-1}\left(\varphi_{0}, I_{0}\right)$ follows from the contraction mapping theorem, under the conditions (6.3) and (6.5) that we have already imposed. To see that, we merely have to show that the following equation has a unique solution $h$ in the space $S$ (of Section 2) for every right side, $\zeta \in S$ :

$$
F_{\varphi} h \equiv \cos \varphi_{0} h-B_{\varphi}\left(\varphi_{0}\right) h=\zeta .
$$

Because of (6.8), this may be written as

$$
h=\left\{1-\left[B\left(\varphi_{0}\right)+I_{0}\right]^{2}\right\}^{-\frac{1}{2}}\left[B_{\varphi}\left(\varphi_{0}\right) h+\zeta\right] \equiv \mathscr{A}(h) .
$$

For all $\zeta \in S$, the operator $\mathscr{A}$ maps $S$ into itself, and the mapping is contractive under conditions (6.3) and (6.5), or (6.6) and (6.5). Hence (6.13) has a unique solution in $S$, for all $\zeta \in S$.

According to Eq. (6.9) we may vary $I$ throughout the region $\Omega$, and still have an amplitude which has the same modulus. This amplitude will obey the constraints of inelastic unitarity, provided we vary $I$ so that the conditions $0 \leqq \eta_{l} \leqq 1$ are respected. It is clear that there is an infinite subset $\Omega^{\prime}$ of $\Omega$ in which these conditions hold. One can obtain the phase of the amplitude, in principle, by solving the differential equation (6.11) with the initial condition (6.19).

The basic problem of phase-shift analysis in the inelastic region may be stated as the determination of $\varphi(x)$ and $I(x)$, given $g(x)$, since this then determines all of the real parts of the phase-shifts and the elasticities. We have already seen that, in the domain where inequalities (6.3) and (6.5) hold, the solution for $\delta_{l}$ and $\eta_{l}$, given $g$, is not unique. In fact, there is a continuum of $\delta_{l}$ 's corresponding to the continuum of $\eta_{l}$ 's for which $I \in \Omega^{\prime}$. Each solution in this continuum gives partial-wave amplitudes $f_{l}$ which lie within the unitarity circle, $\left|1+2 i f_{l}\right| \leqq 1$.

From the implicit function theorem alone, one does not have a useful estimate of the size of the region $\Omega$. One expects that the solution of the differential equation (iii) will extend at least as far as the first singularity of $F_{\varphi}$, but the location of such singularities is a quantitative question. In specific cases, one might throw some light on the problem by numerical solution of the differential equation [5]. For numerical work, it would be appropriate to effect the alteration in $I$ by varying a finite number of the $\eta_{l}$. In the simplest situation we would vary only one $\eta_{l}$ at a time, in which case the operator differential equation (6.11) is replaced by the vector differential equation

$$
\frac{d \varphi}{d \eta_{l}}=-F_{\varphi}^{-1}\left(\varphi\left(\eta_{l}\right)\right) \frac{1}{g}\left(l+\frac{1}{2}\right) \eta_{l} P_{l} .
$$

In practical phase-shift analysis, one normally introduces the constraint of the optical theorem; i.e.,

$$
\operatorname{Im} f(1)=\frac{k^{2}}{4 \pi} \sigma
$$


where $\sigma$ is the total cross-section. In the elastic case, the solution of the integral equation (2.2) automatically satisfies Eq. (6.15). In the inelastic case, the optical theorem determines $I(1)$ in terms of $\sigma$ and $\sigma_{\varepsilon l}$, the latter being the total elastic cross-section. By evaluation of Eq. (6.1) at $x=1$, one obtains

or

$$
\operatorname{Im} f(1)=\frac{1}{4 \pi} \int_{-1}^{1} d y \int_{0}^{2 \pi} d w g^{2}(y)+g(1) I(1)
$$

$$
\frac{k^{2}}{4 \pi} \sigma=\frac{k^{2}}{4 \pi} \sigma_{\varepsilon l}+g(1) I(1) \text {. }
$$

In varying the $\eta_{l}$ for a given $g(x)$ and given $\sigma$, we must then observe the constraint

$$
\sum_{l=0}^{\infty}(2 l+1)\left(\frac{1-\eta_{l}^{2}}{4}\right)=\frac{k^{2}}{4 \pi}\left(\sigma-\sigma_{\varepsilon l}\right) .
$$

In the above argument concerning variations of the inelastic term, we are now restricted to variations of $I$ within a set $\Omega^{\prime \prime} \subset \Omega^{\prime} \subset \Omega$. We could easily permit the quantities $\eta_{l}$ to vary, such that the constraint (6.17) is satisfied. Clearly, this still allows continuous variations of $I$, so we retain the continuum ambiguity in inelastic phase-shift analysis.

\section{Appendix}

An easy improvement of Theorem 1 is possible, since

$$
\sin v=\inf _{-1 \leqq x \leqq 1} \iint H(x, y, z) d y d z
$$

is necessarily greater than zero. The set

$$
\tilde{T}=\{\varphi: \varphi \in S, \lambda \leqq \varphi \leqq \mu\}, \quad \lambda>0, \quad \mu<\pi / 2 .
$$

is mapped by $A$ into itself, if

$$
\tan \lambda \leqq \tan \lambda_{0}=\frac{\sin v \cos \mu}{1-\sin v \sin \mu} .
$$

This is seen by noting that if $\varphi \in \tilde{T}$, then

$$
\sin \nu \cos (\mu-\lambda) \leqq \sin A(\varphi) \leqq \sin \mu .
$$

From (2.15) one has that

$$
\left\|A^{\prime}(\varphi) h\right\| \leqq \frac{2 \sin \mu \sin (\mu-\lambda)}{\left(1-\sin ^{2} \mu\right)^{\frac{1}{2}}}\|h\|, \quad \varphi \in \tilde{T}, \quad h \in S,
$$

so that $A$ is a contraction mapping of $\tilde{T}$ into itself provided that

$$
2 \tan \mu \sin (\mu-\lambda)<1 \text {. }
$$


If $0 \leqq \mu \leqq \mu_{1}$, we retrieve the results of our previous discussion, since then both (A.6) and (A.3) are satisfied with $\lambda=0$. If $\mu_{1} \leqq \mu<\pi / 2$, inequality (A.6) requires $\lambda_{1}<\lambda<\lambda_{2}$, where $\lambda_{1}$ and $\lambda_{2}$ are the two roots of the equation

$$
2 \tan \mu \sin (\mu-\lambda)=1 .
$$

This equation is quadratic in $\tan \lambda$, and has the solutions

$$
\tan \lambda=\cos \mu \frac{(2 \tan \mu \sin \mu)^{2}-1}{4 \sin ^{3} \mu \pm\left[5 \sin ^{2} \mu-1\right]^{\frac{1}{2}}} .
$$

The solutions are both positive if $\mu_{1}<\mu<\pi / 2$, while $\lambda_{1}\left(\mu_{1}\right)=0$. Thus, the condition $\lambda>\lambda_{1}$ joins continuously to the condition $\lambda=0$ as $\mu$ passes through $\mu_{1}$. To apply the contraction mapping principle for $\mu_{1} \leqq \mu<\pi / 2$, we require $\tan \lambda_{1}<\tan \lambda$ in order to satisfy (A.6), and also $\tan \lambda \leqq \tan \lambda_{0}$ to satisfy (A.3). Since $\tan \lambda_{0}$ is a monotonic increasing function of $\sin v$, the resulting inequality $\tan \lambda_{1}<\tan \lambda_{0}$ implies a lower bound on $\sin v$. The explicit lower bound may be read off from (A.8) and (A.3), but it comes out in a simpler form through the equivalent procedure of solving the following equation for $\sin v$ :

$$
2 \tan \mu \sin \left(\mu-\lambda_{0}(\sin v, \mu)\right)=1 .
$$

This is quadratic in $\sin v$, and leads to the result

$$
\sin v>\sin \mu-\left(4 \tan ^{2} \mu-1\right)^{-\frac{1}{2}} \cos \mu, \quad \mu_{1} \leqq \mu<\pi / 2 .
$$

In Fig. 3 we show a graph of the right hand side of (A.10). Because $\sin v>0$, we always obtain a bigger range of $\mu$ than that allowed in Theorem 1.

Theorem 1 A. If $g(x)$ is continuous on $[-1,1], \mu_{1} \leqq \mu<\pi / 2$, and $\sin v>\sin \mu-\left(4 \tan ^{2} \mu-1\right)^{-\frac{1}{2}} \cos \mu$, there is a unique continuous solution

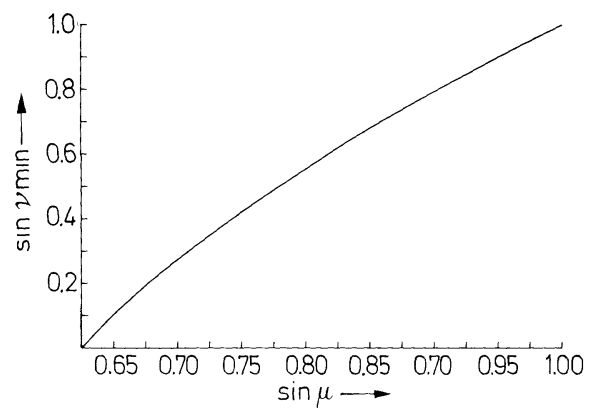

Fig. 3. The minimum of $\sin v$, as a function of $\sin \mu$ 
$\varphi$ with $0 \leqq \lambda \leqq \varphi \leqq \mu$; here $\lambda_{1}<\lambda<\lambda_{2}$, where $\lambda_{1}$ and $\lambda_{2}$ are given by Eq. (A.8). The solution is the limit of the sequence $\left\{\varphi_{n}\right\}$, where $\varphi_{n+1}=A\left(\varphi_{n}\right)$, and $\varphi_{0}$ is any continuous function such that $\lambda \leqq \varphi \leqq \mu$.

The advantage of Theorem 1A over Theorem 1 becomes more important as the upper and lower bounds of the integral $\iint H(x, y, z) d y d z$ draw together. In the extreme event that this integral is constant, (realized in the case of pure $s$-wave scattering where $g(x)=g=$ constant), the hypotheses of Theorem 1A are satisfied for any $\mu<\pi / 2$. We then have $\varphi(x)=\sin ^{-1} g=\mu$ as the unique continuous solution for which $-\pi / 2 \leqq \varphi(x) \leqq \pi / 2$. This result was proved to one of us in a slightly different way by Martin.

Acknowledgments. We wish to thank G. R. Bart, A. Martin, and R. G. Newton for helpful conversations and correspondence. R. L. Warnock enjoyed the hospitality of the Physikalisches Institut of the University of Bonn and the Department of Physics of Imperial College during the course of this work.

\section{References}

1. Crichton, J.H.: Nuovo Cimento 45 A, 256 (1966).

2. Newton, R. G.: J. Math. Phys. 9, 2050 (1968).

3. Martin, A.: Nuovo Cimento 59 A. 131 (1969).

4. Gerber, R. B., Karplus, M.: Phys. Rev. D 1, 998 (1970);

Goldberg, H.: Phys. Rev. D 1, 1242 (1970);

Sakmar, I. A.: Lett Nuovo Cimento 2, 256 (1969);

Eftimiu, C.: J. Math. Phys. 12, 2047 (1971);

Bowcock, J.E., Hodgson, D. C.: (Birmingham preprint);

Burkhardt, H.: (Birmingham preprint);

Gersten, A. : Nucl. Phys. B 12, 537 (1969);

Klepikov, N. P.: JETP 41, 1187 (1961), trans. 14, 846 (1962);

Wageningen, R. van: Thesis, Groningen (1957)

5. Specific techniques for numerical solution of the integral equation are discussed by R. L. Warnock. New Methods for Numerical Solution of Nonlinear Equations, with Suggested Applications in S Matrix Theory, notes of lectures at the University of Bonn, February, 1972, submitted for publication in Fortschritte der Physik.

6. Dieudonné,J.: Foundations of Modern Analysis. New York: Academic Press 1960.

7. Krasnosel'skii, M.A.: Topological Methods in the Theory of Nonlinear Integral Equations, p. 124. New York: MacMillan 1964.

D. Atkinson

University of Groningen

Institute for Theoretical Physics

P.O. Box 800

Groningen, The Netherlands 